\title{
RF Technologies for Advancing Space Communication Infrastructure
}

\author{
Irene Bibyk, (216) 433-6708, Irene.K.Bibyk@nasa.gov, \\ Robert Romanofsky, (216) 433-3507, Robert.R.Romanofsky@nasa.gov, \& \\ Ed Wintucky, (216) 433-3510, Edwin.G.Wintucky@nasa.gov \\ NASA Glenn Research Center \\ 21000 Brookpark Road \\ Cleveland, OH 44135
}

\begin{abstract}
This paper ${ }^{12}$ will address key technologies under development at the NASA Glenn Research Center designed to provide architecture-level impacts. Specifically, we will describe deployable antennas, a new type of phased array antenna and novel power amplifiers. The evaluation of architectural influence can be conducted from two perspectives where said architecture can be analyzed from either the "top-down" to determine the areas where technology improvements will be most beneficial or from the "bottom-up" where each technology's performance advancement can affect the overall architecture's performance. This paper will take the latter approach with focus on some technology improvement challenges and address architecture impacts. For example, using data rate as a performance metric, future exploration scenarios are expected to demand data rates possibly exceeding $1 \mathrm{Gbps}$. To support these advancements in a Mars scenario, as an example, Ka-band and antenna aperture sizes on the order of 10 meters will be required from Mars areostationary platforms. Key technical challenges for a large deployable antenna include maximizing the ratio of deployed-topackaged volume, minimizing aerial density, maintaining RMS surface accuracy to within $1 / 20$ of a wavelength or better, and developing reflector rigidization techniques. Moreover, the high frequencies and large apertures manifest a new problem for microwave engineers that are familiar to optical communications specialists: pointing. The fine beam widths and long ranges dictate the need for electronic or mechanical feed articulation to compensate for spacecraft attitude control limitations.
\end{abstract}

\section{TABLE OF CONTENTS}

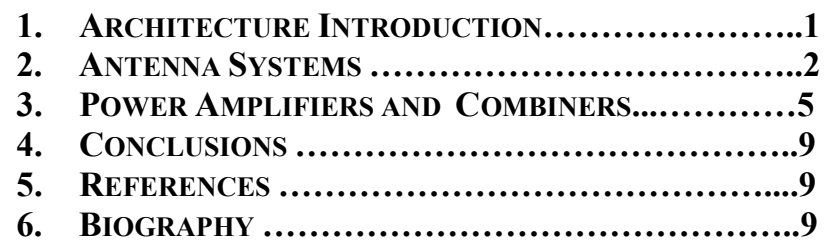

\footnotetext{
${ }^{1}$ 0-7803-9546-8/06/\$20.00@ 2006 IEEE

${ }^{2}$ IEEEAC paper \#1552, Version 6, Updated Jan, 112005
}

\section{ARCHITECTURE INTRODUCTION}

Communications requirements derived from the human and robotic exploration vision dictate a sophisticated, layered architecture that is dramatically different than what was required for the low-rate, point-to-point communications of the Apollo era. Emerging but relatively near-term exploration initiative needs include: tele-operation and autonomous robotic missions, lunar reconnaissance and orbiting relay satellites, cooperative spacecraft, lunar surface wireless local area networks, extremely wide bandwidth links to support hyper-spectral imaging, synthetic aperture radar, etc., and other modern features such as high definition television and tele-medicine. For example, it has been proposed that data rates on the order of 1 Gbps will be required from Mars areostationary relay satellites. Such enormous data rates and extreme link distances can lead to 10 meter class microwave antennas, likely operating at Ka-band frequencies. The choice of frequency is driven by multiple conflicting requirements. In addition to identifying allocated spectrum with sufficient bandwidth, there may be interference issues to consider. For example, Earth-based fixed satellite service uplinks at $\mathrm{Ku}$-band and terrestrial radar at S-band may pose a problem in the vicinity of the moon. And the radio astronomy community is concerned with preserving future observatories in the shielded zone of the moon. An acceptable candidate for lunar orbiter-to-lunar surface and vice-versa high data rate links is 25.5 to $27 \mathrm{GHz}$. This band is also expected to be acceptable for lunar orbiter-to-Earth communications. Likely return links from Mars will be $\sim 32$ or $\sim 38 \mathrm{GHz}$.

As previously mentioned, the architecture for lunar exploration is expected to involve a layered communications and navigation network. This network may include lunar vicinity relay satellites at L1 and L2 Lagrange points as well as lunar polar orbiting satellites. The lunar proximity network must be able to access dedicated assets such as Malapert Station and eventually include human assets, such as crewed rovers, as relay nodes. We postulate that mission science data from topographic and spectral measurements, coupled with robotic tele-operation and telepresence and eventual physical human presence, may accumulate to terabits-per-day. In addition, a wide variety of surface and orbiting assets must be supported simultaneously. The architecture that ultimately evolves must provide a solution 
that emphasizes asset networking and the safety of 24/7 communications back to Earth. Intuitively, improvements of antenna and power amplifiers have the most significant impact on the architecture-level performance. These improvements translate to more data with less power and mass, which are two essential constraints on in-space systems.

\section{ANTENNA SYSTEMS}

\section{Large Deployable Technology}

Critical performance specifications for these very large antennas include: low aerial density $\left(\sim 1 \mathrm{~kg} / \mathrm{m}^{2}\right)$, high packaging efficiency (i.e. ratio of deployed to stowed volume $>5: 1$ ), accurate surface geometry to ensure high reflector efficiency (rms surface errors $<\lambda / 20$ ), reliable deployment and stability. NASA's Glenn Research Center (GRC) is currently working on several approaches including inflatable membranes with a rigidized torus and a hybrid shape-memory composite approach that incorporates a fixed central reflector that serves as a backup antenna in case of deployment problems with the main aperture. GRC is partnering with SRS Technologies (Huntsville, AL) on the former and Johns Hopkins University (Laurel, MD)/ ILC Dover (Frederica, DE) on the latter. The goal is to develop large gossamer antennas for deep space and lunar/Mars applications that have the potential to reduce mass, lower cost, and improve deployment reliability while maintaining very accurate surface tolerances over long deployed durations.

GRC and SRS Technologies developed a 4 meter by 6 meter offset parabolic inflatable membrane reflector as shown in Figure 1. Using an off-the-shelf feed, a gain of $\sim 48 \mathrm{~dB}$ was measured at $8.4 \mathrm{GHz}$ in the GRC near field range and corresponds to a $\sim 52 \%$ efficiency when compared with an ideal antenna. Considering that the calculated directivity was $49.7 \mathrm{~dB}$ and the measured directivity was $48.0 \mathrm{~dB}$, a fair estimate of actual efficiency is $\sim 67 \%$. A customized corrugated feed that provides an $11 \mathrm{~dB}$ aperture taper is expected to yield an aperture efficiency of $75 \%$ and a peak directivity of $50.1 \mathrm{~dB}$. Phase plots of the aperture more or less agree with the measured $3.5 \mathrm{~mm}$ average surface shape accuracy of the surface. The extreme antenna edges show some significant phase changes that are implicit with an inflatable lenticular geometry (i.e. flattened edges). Surface shape accuracy can be improved through inflation pressure adjustment and/or catenary tension adjustment. This type of antenna is expected to achieve a packaging efficiency greater than 50:1.

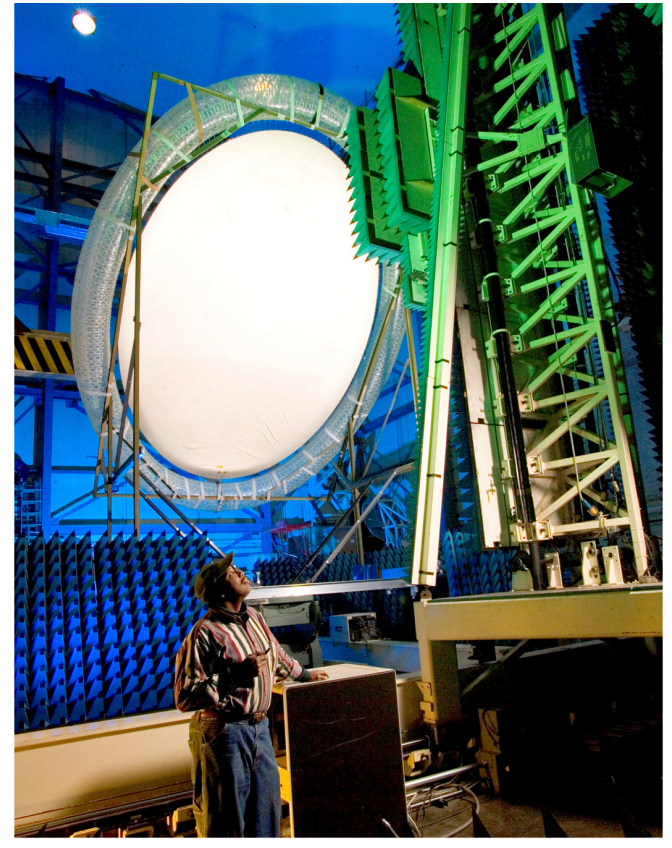

Figure 1 - SRS 4 x 6 meter inflatable reflector in GRC's near-field antenna range.

The shape memory antenna system combines a fixed parabolic dish with an inflatable reflector annulus [2]. This antenna, based on a novel shape memory composite structure, provides scaleable high gain antenna architecture.

Other types of deployable antennas under consideration are mesh reflectors, such as the radial-rib or "umbrella" type as well as the tensioned truss concept. Potential problems to be overcome for Ka-band operation include mesh reflectivity (and leakage) and faceting errors. It has been shown [3] that the maximum achievable gain is proportional to $\mathrm{D}^{2} / \varepsilon^{2}$, where $D$ is antenna diameter and $\varepsilon$ is the rms surface tolerance error representing deviation of a facet from spherical. i.e. the local radius of curvature is spherical, an approximation to the actual parabolic geometry. To minimize $\varepsilon$ the number of triangular facets, or equivalently the number of nodes $\mathrm{N}$, in the mesh reflector must be increased as frequency increases. Note that $\mathrm{D} / \varepsilon$ is proportional to $\mathrm{N}$ so gain is proportional to $\mathrm{N}^{2}$. This trend suggests that mass or aerial density increases with frequency. Nevertheless this type of reflector system has already proven itself via several successful commercial satellite systems at $\mathrm{S}$ - and L-band.

It is anticipated that the greatest challenge for any style antenna system will be achieving the required Ka-band surface accuracies. And, reliable deployment from a packaged state continues to be an area of risk. On-orbit rigidization remains somewhat of an issue with regard to inflatable membranes and response in the natural thermal environment. 


\section{Ferroelectric Reflectarray Antenna}

A scanning reflectarray consists of a flat surface with diameter $D$ containing $N$ integrated phase shifters and $N$ patch radiators that is illuminated by a single feed at a virtual focus located a distance $f$ from the surface such that $f / D \approx 1$. The modulated signal from the feed passes through the $N$ reflect-mode phase shifters and is re-radiated as a focused beam in essentially any preferred direction in the hemisphere in front of the antenna, as in a conventional phased array. The control algorithm is nearly identical to that of a conventional phased array, with the exception of an a priori setting of all phase shifters to compensate for the spherical wave-front from the feed. That is, the signal from the feed reaches the central element of the aperture before it reaches elements toward the perimeter. The physics insofar as inter-element spacing, mutual coupling, scan loss, etc. is concerned is the same as for a conventional, directly radiating phased array. A photograph of a prototype ferroelectric reflectarray is shown in Figure 2.

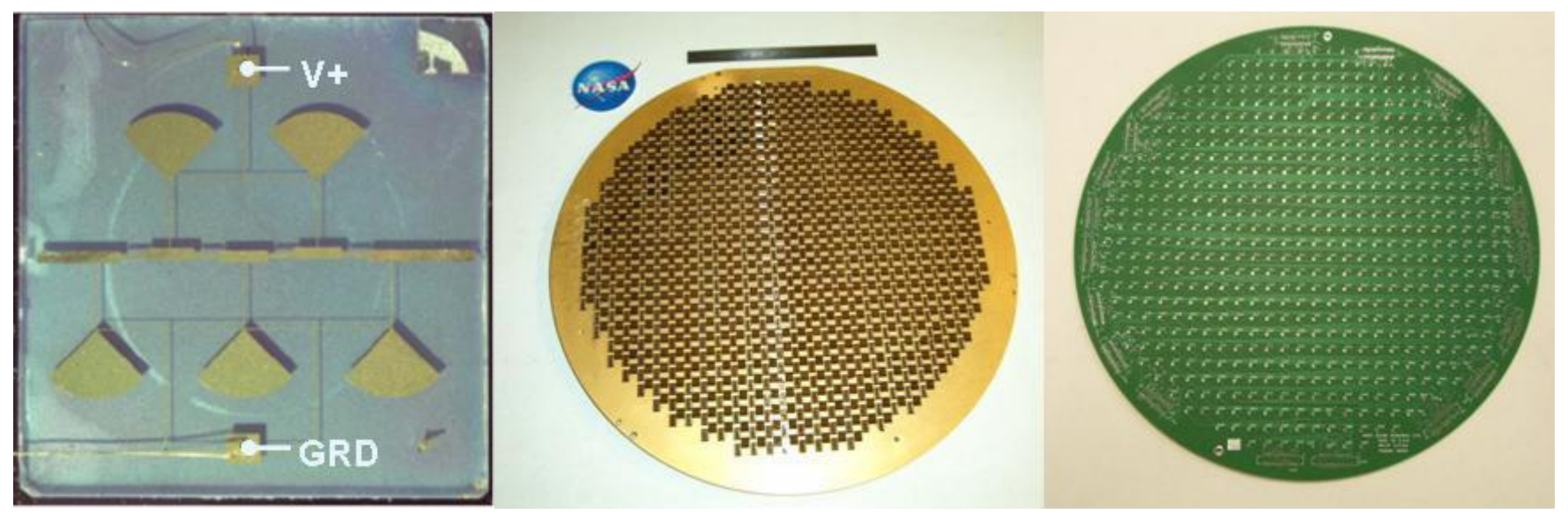

Figure 2 - Partial anatomy of prototype K-band scanning reflectarray antenna incorporating 615 thin film ferroelectric phase shifters (left). Each phase shifter is wire bonded to microstrip patch antenna. The active array diameter (center) is $31 \mathrm{~cm}$. The 6-layer bias control board engages 615 bias pins on the backside of the reflectarray platen. The associated controller consumes $22 \mathrm{~W}$. A dual-mode feed horn (not shown) illuminates the array. $\mathrm{F} / \mathrm{D}=1$.

The primary advantage of a reflectarray is power efficiency. This efficiency stems from the elimination of a beam forming manifold because of the quasi-optical feed. Now, to a first order, gain is proportional to the number of elements. However, in a conventional phased array, gain is actually asymptotic to some value. That value is determined when manifold transmission line loss overcomes gain increase from adding more elements. Free of this limitation, the reflectarray can, in principle, generate any EIRP since the aperture can become arbitrarily large.

Moreover, a single amplifier with modest power can be used to drive the system. A comparison of a reflectarray and a conventional "directly radiating" phased array is provided in Figure 3. Manifold loss in the MMIC array is evident. 


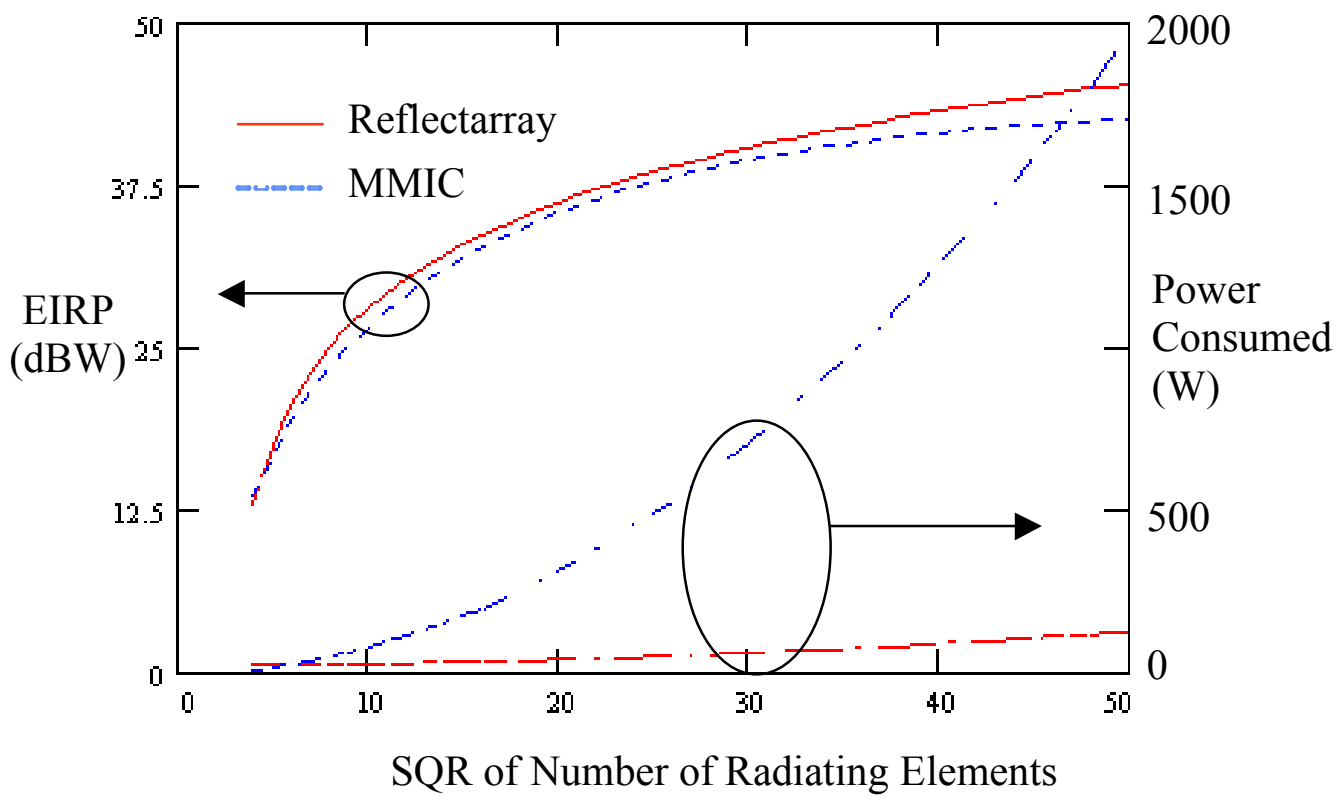

Figure 3 - Calculated EIRP and power consumption for a ferroelectric reflectarray and a direct radiating MMIC phased array at Ka-band as a function of the square root of the number of radiating elements. Reflectarray Assumptions: $10 \mathrm{~W}, 40 \%$ efficient TWT, $4 \mathrm{~dB}$ loss phase shifters, $41 \mathrm{~mW}$ per channel controller power consumption. Direct Radiating MMIC Array Assumptions: $100 \mathrm{~mW}, 15 \%$ efficient amplifiers, $85 \%$ efficient power supply.

One difficulty with implementing the reflectarray arises since the phase shifters are between the feed horn and the patch radiating elements. As a result, in a receive array, they introduce line loss in front of the first stage low noise amplifier (LNA) and cause system noise temperature to escalate. Similarly, in the case of a transmit array, they dominate efficiency since they follow the power amplifier. Figure 4 shows calculated noise temperature as a function of phase shifter loss. Constructing the active array economically can be a challenge. We have devised very low loss phase shifters based on thin ferroelectric films [45]. Moreover, the phase shifters require only one bias line and can be built using a simple lithography process since the smallest feature size is on the order of $10 \mu \mathrm{m}$. Another reason to believe that the reflectarray will have profound cost advantages is attributed to its simple structure. The reflectarray requires only a multilayer dc bias distribution board, a support platen that also serves as the DC and RF ground plane, and the RF layer populated with $2 N$ devices that can be automatically placed and wire bonded. The system's front end consists of a corrugated or dual-mode feed horn plus the supporting struts, an LNA, and a controller.

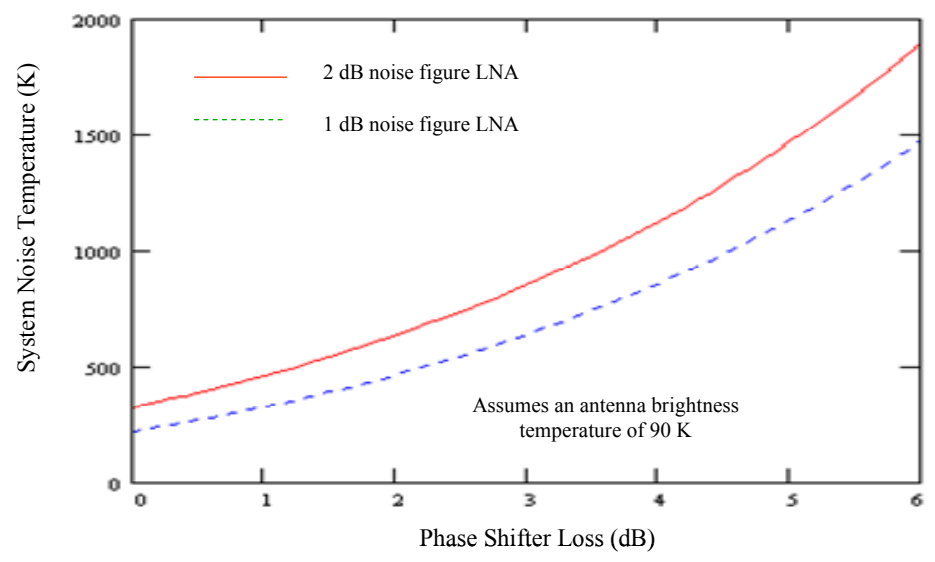

Figure 4 - Calculated reflectarray system noise temperature as a function of phase shifter insertion loss. 
We developed X-band phase shifters that consist of four cascaded coupled microstrip lines patterned over $400 \mathrm{~nm}$ thick laser ablated $\mathrm{Ba}_{0.50} \mathrm{Sr}_{0.50} \mathrm{TiO}_{3}$ films, which is followed by a switch. The "ferroelectric" section provides nominally 180 degrees of analog phase shift. As a bias from 0 to 350 $\mathrm{V}$ is applied across the coupled line electrodes, the relative dielectric constant of the film tunes from about 2000 to 800, thereby modifying the propagation constant. Note that the ferroelectric films are excellent dielectrics and the current draw is negligible so there is virtually no power consumption. The beam lead GaAs diode switch toggles between an open and a virtual short circuit realized with a quarter-wave radial stub.

This results in a digital transition between a reflection coefficient with magnitude near unity and phase of $\approx 0$ degrees and $\approx 180$ degrees, respectively. Thus, a full $2 \pi$ phase shift is possible. Prototype X-band phase shifters exhibited less than $3 \mathrm{~dB}$ insertion loss. An insertion loss $<$ $4 \mathrm{~dB}$ is anticipated at Ka-band.

\section{Pointing Loss}

The large aperture antennas at Ka-band previously described will have correspondingly small beamwidths. For example, at $32 \mathrm{GHz}$ the beamwidths of a $10 \mathrm{~m}$ and $5 \mathrm{~m}$ aperture are about $0.070^{\circ}$ and $0.130^{\circ}$, respectively. Calculated values of pointing loss as a function of pointing error are illustrated in Figure 5. This paper will not address the current capabilities of spacecraft attitude control systems. However, at Ka-band and especially with large apertures, some type of active fine beam pointing may be required to compensate for the stability challenges. A gimbal system to manipulate the entire structure may not be practical because of speed and induced vibrations. To achieve beam steering on the order of a few beamwidths, it is more likely that some type of articulated feed will be used. A mechanically articulated feed, a cluster of feed horns, active phased array feed, and a scanning reflectarray are being considered. For example, the scanning reflectarray could be used as the subreflector in a Cassegrain geometry. Its primary purpose would be to emulate a hyperbolic surface and then to redirect the feed signal to a slightly off-axis position to effect subtle steering. While this type of scanning results in some loss $(<1 \mathrm{~dB})$ due to defocusing of the feed, it is a necessary tradeoff considering the potentially catastrophic result of only coarse pointing with spacecraft attitude control.

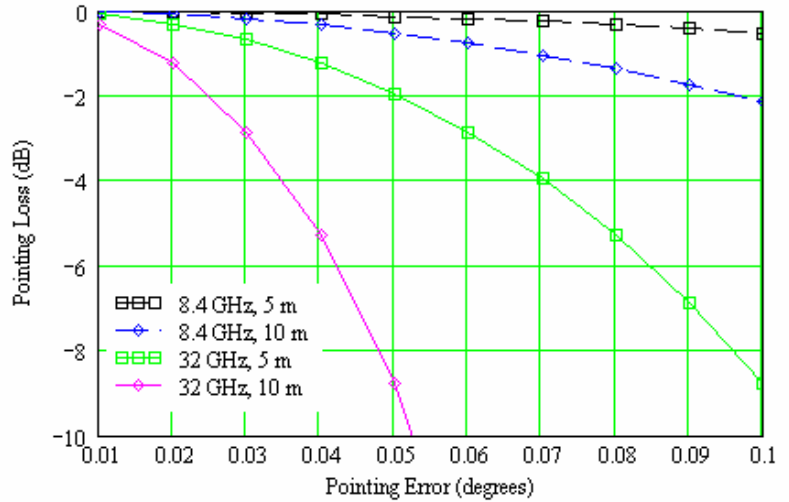

Figure 5 - Calculated pointing loss as a function of pointing error at X- and Ka-band for 5 and 10 meter antenna assuming a circular aperture and an $11 \mathrm{~dB}$ edge taper.

\section{POWER AMPLIFIERS AND COMBINERS}

\section{Power Amplifiers}

Along with larger antenna and higher carrier frequencies, higher power amplifiers are needed for higher data rates and can be found in several forms as a Traveling Wave Tube Amplifier (TWTA), Solid State Power Amplifier (SSPA) or as a combination of several single devices to increase the total RF power output. GRC designed a high efficiency, miniaturized, Ka-band TWT based on a novel, high gain "finned ladder" RF/electron beam interaction circuit and small diameter $(1.25 \mathrm{~mm})$ electrically efficient $(1 \mathrm{~W})$ barium dispenser thermionic cathode that can be incorporated into the reflectarray at the feed to enable a new benchmark in phased array efficiency. As ascertained from Figure 3, with the reflectarray, arbitrarily high EIRP can be achieved by increasing the number of array elements instead of increasing amplifier power. Whereas solid state power amplifiers have historically been used in low power (i.e. $\sim$ several watts) applications, traveling wave tubes excel at power levels on the order of $100 \mathrm{~W}$. An optimal drive level for the reflectarray involves a trade between efficiency, aperture size, and intersymbol interference effects. The trade analysis resulted with an optimal drive level around 10 W, where neither SSPAs nor TWTs can claim dominance. The following table is a comparison of the "mini-TWT" and commercially available SSPAs.

Investigations of $\mathrm{GaN}$ devices continue to show promise although considerable challenges continue with material reliability. For even higher output powers as will be required for high data rate deep-space links, the next section will describe TWTA power combiner work that has recently been completed at GRC. 
Table 1 - Comparison of Ka-band $10 \mathrm{~W}$ class vacuum tube and commercially available solid state power amplifiers

\begin{tabular}{|c|c|c|}
\hline & $\begin{array}{c}\text { Miniature TWT } \\
\text { (under development) }\end{array}$ & SSPA \\
\hline $\begin{array}{c}\text { Efficiency } \\
\mathrm{P}_{(1 \mathrm{~dB})}\end{array}$ & $50 \%$ (design goal) & $10-12.5$ \\
\hline Bandwidth & $\begin{array}{c}500 \mathrm{MHz} \text { (design goal), } \\
\min 200 \mathrm{MHz}\end{array}$ & $200 \mathrm{MHz}$ to $2 \mathrm{GHz}$ \\
\hline Gain & $\begin{array}{c}40 \mathrm{~dB} \text { (present design - } \\
\text { easily made larger) }\end{array}$ & 40 to $50 \mathrm{~dB}$ \\
\hline Dimensions & $\begin{array}{l}\text { 3" length x } 0.5 \text { " dia } \\
\text { (suitable for feed } \\
\text { mount, minimal } \\
\text { blockage) }\end{array}$ & $\begin{array}{c}7.6 " \times 3.5 " \times 3.25 " \\
\text { to } \\
9 " \times 6 " \times 1.75 " \text { (not } \\
\text { suitable for feed } \\
\text { mount) }\end{array}$ \\
\hline Weight & $\begin{array}{c}500 \mathrm{~g} \max (1.1 \mathrm{lb}) \\
\text { EPC mounted remotely }\end{array}$ & 2.5 to $3 \mathrm{lb}$ \\
\hline TRL & 2 & 6 \\
\hline
\end{tabular}

\section{Power Combiners}

Deep space missions that require data transmission rates of 1 Gbps (i.e. the Mars-to Earth data link) will also require transmitter powers in the range of 1 to $10 \mathrm{~kW}$. Since these high powers are well above the limits of single TWTAs, some form of power combining will be required. One feasible approach is the magic-T based wave guide power combiner, which is the preferred approach currently flown in commercial communications satellites with high combining efficiency $(<0.1 \mathrm{~dB}$ per magic-T) and powers of at least $2 \mathrm{~kW}$ at $\mathrm{C}$-band and $\mathrm{Ku}$-bands. The key to successful operation of this high power combining approach is the magic-T (a 4-port wave guide hybrid junction), the basic configuration of which is shown in Figure 6 . The magic- $T$ is designed so that the input signals add in phase at one output (port 2 in Figure 6) and cancel in phase at the other output (port 3 in Figure 6). Optimum performance as a power combiner therefore requires a balance of powers and phases at the two input ports. The magic-T allows efficient combining of the TWTAs in pairs as shown in Figure 7. A back-up TWT can be added and easily switched into operation if needed for greater reliability.

The feasibility of the wave guide magic-T power combining approach at Ka-band was successfully demonstrated at GRC for two cases at or near saturated power in a two-way combiner. One case was with two 110-115 W ACTS TWTAs at 29.1 to $29.6 \mathrm{GHz}$ and the second with two high efficiency $100 \mathrm{~W}$ space TWTs (Boeing 999H) over the DSN frequency band of 31.8 to $32.3 \mathrm{GHz}$. The power combiner test bed for the two $32 \mathrm{GHz} 100 \mathrm{~W}$ TWTs is shown in Figure 8 . Power combining efficiencies were in the range of $90 \%$ to $95 \%$ (depending on the frequency) using a commercial off-the-shelf magic-T. Computer modeling at GRC of magic-T and alternative hybrid junction designs for low loss and high power handling capability showed combining efficiencies $>95 \%$ were achievable. High efficiency, higher power TWTs will be available for power combining applications. The $100 \mathrm{~W}$ TWT has been upgraded to $180 \mathrm{~W}$ while $250 \mathrm{~W}$ has also been demonstrated. An appropriate modification of the wave guide circuit to equalize the rates of change of phase with frequency at the two input ports extended the useful bandwidth of the magic-T to that of the individual TWTs. This modification makes a data transmission rate of $1 \mathrm{Gbps}$ or more potentially possible. This is shown in the power comparison in Figure 9 for a $1 \mathrm{GHz}$ frequency band. A data transmission rate through the magic-T of $622 \mathrm{Mbps}$ (QPSK) with a signal bandwidth of $311 \mathrm{MHz}$ and a low bit error rate of $2.4 \times 10^{-8}$ was successfully demonstrated. 


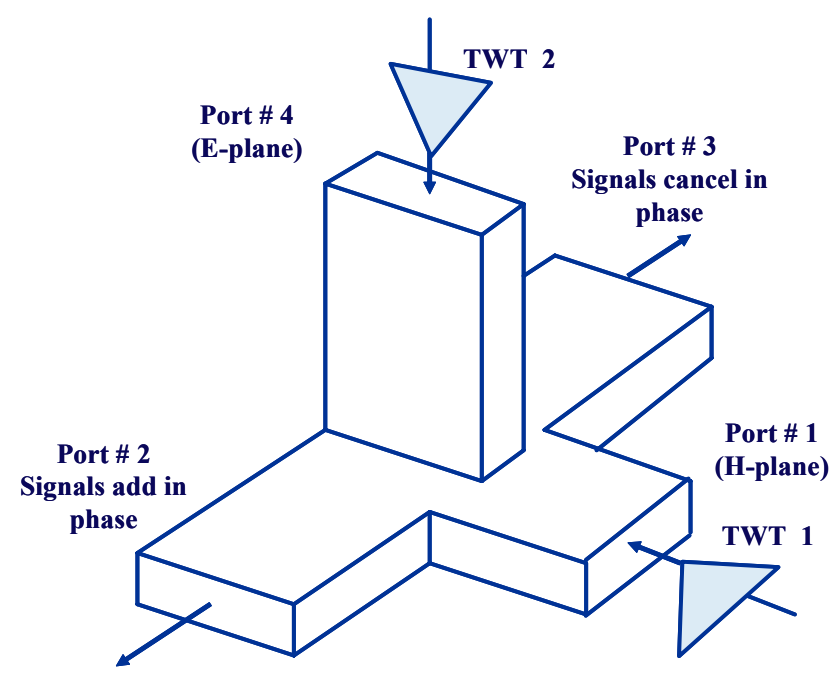

Figure 6 - Basic configuration of wave guide magic-T as a combiner for two microwave TWTs.

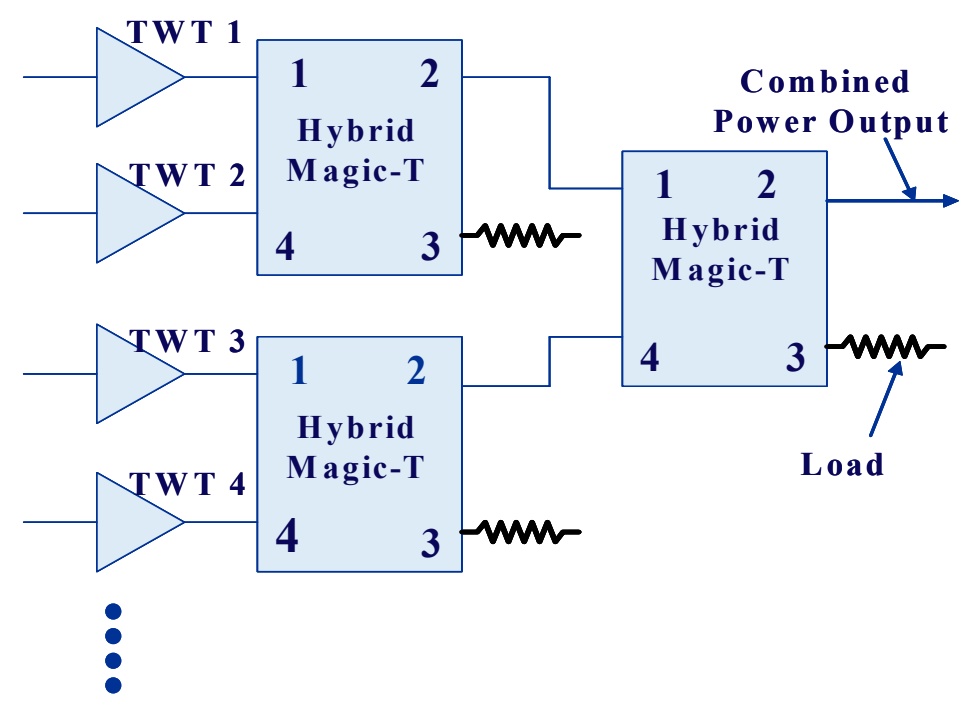

Figure 7 - Binary circuit architecture for combining $2^{\mathrm{n}}$ TWTs where $\mathrm{n}$ is an integer. 


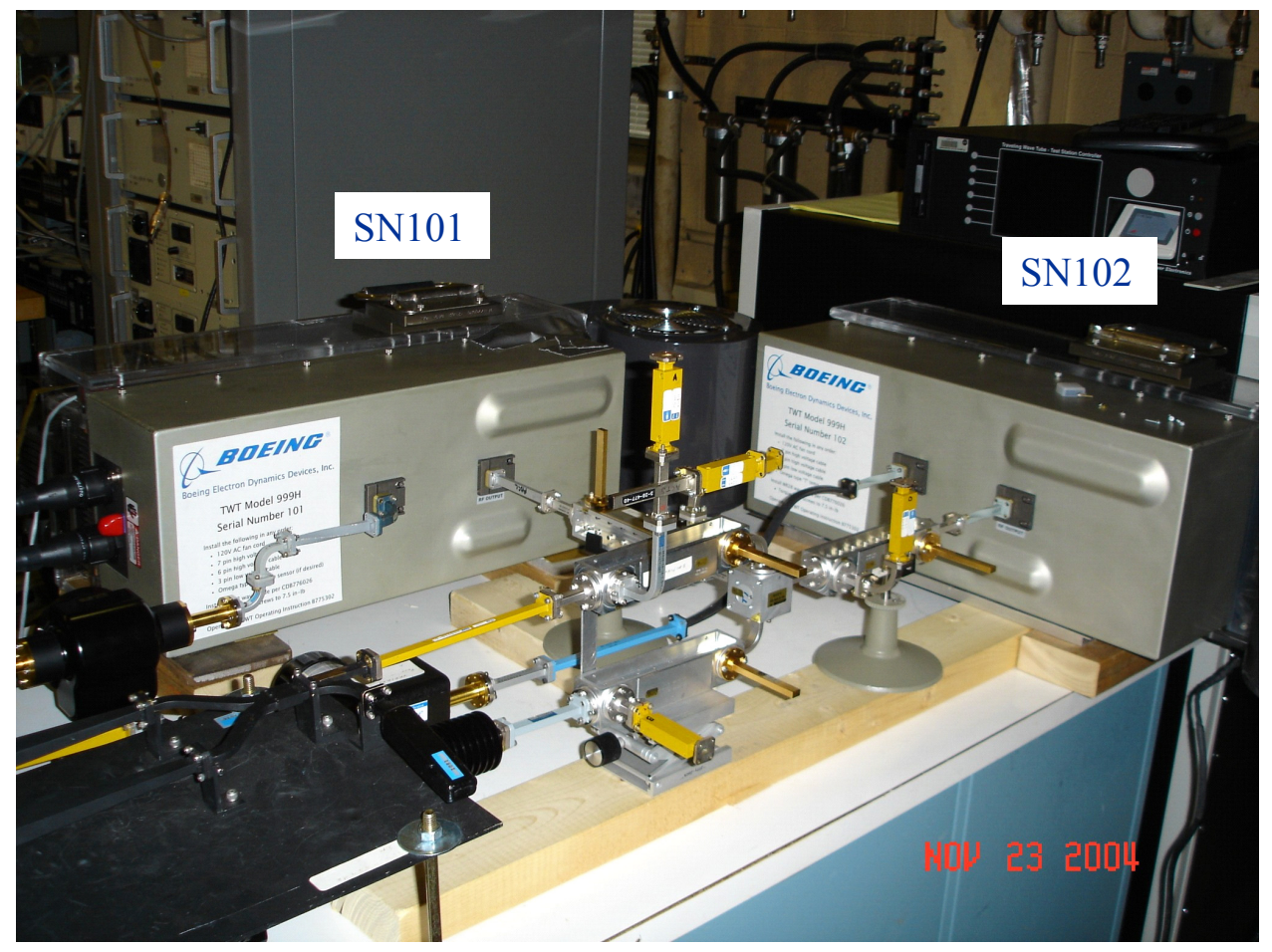

Figure 8 - Power combining test bed for two $32 \mathrm{GHz} 100 \mathrm{~W}$ TWTs.

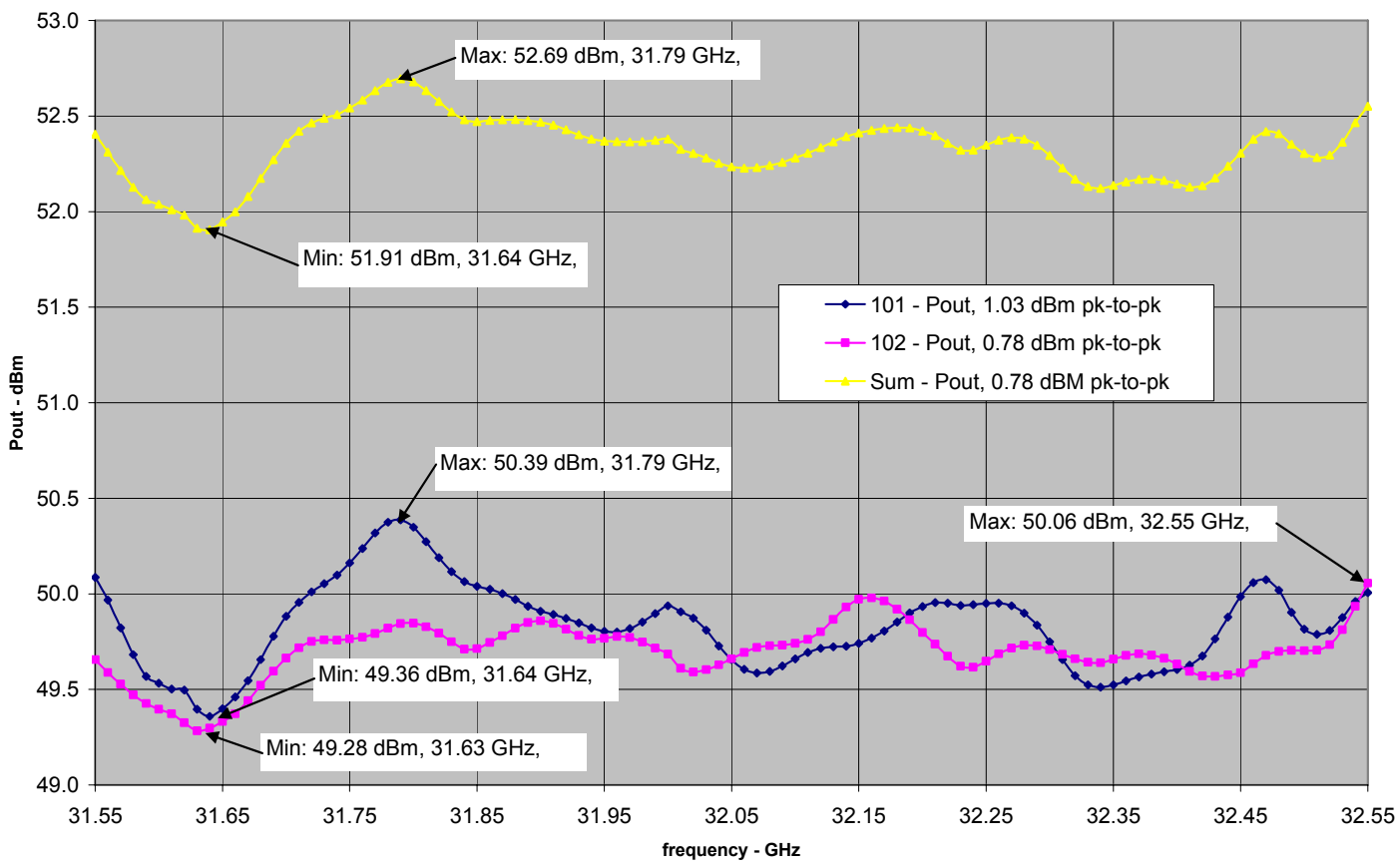

Figure 9 - Power comparison of combined power with individual TWT powers over a 1 $\mathrm{GHz}$ frequency band showing useful bandwidth of magic-T. 


\section{Conclusions}

This paper addresses several key technologies under development at GRC to address future space communication needs including: large deployable antenna, power amplifiers and novel scanning phased array antenna. The need for Ka-band frequencies to support the envisioned data rates places a considerable burden on these new technologies. For example, maintaining the high surface accuracies $(<\lambda / 20)$ over large apertures represents a considerable technical challenge. Also, the large apertures equate to narrow beamwidths necessitating fine beam pointing. GRC's ferroelectric reflectarray holds promise to provide one possible solution for certain antenna geometries. With the current state of technology, TWTAs have an advantage over SSPAs in terms of efficiency and consequently are receiving a lot of attention for the new architectures. Power combining several TWTs to achieve the requisite power levels to support GBPS data rates continues to be an area of development.

\section{REFERENCES}

1. "Thin Film Antenna Development and Optimization", J. Pearson and R. Romanofsky, AIAA/ASME/AHS Adaptive Structures Conference, Newport, RI , May 1-4, 2006.

2. "A Hybrid Inflatable Dish Antenna System for Spacecraft," C. Willey, R. Schulze, R. Bokulic, et al., 42nd AIAA/ASME/ASCE/AHS/ASC Structures, Structural Dynamics, and Materials Conference, April 16-19, 2001, Seattle, WA.

3. "Electrostatically Figured Reflecting Membrane Antennas for Satellites," J. Lang and D. Staelin, IEEE Trans. Automatic Control, Vol. AC-27, No. 3, pp. 666 670, June, 1982.

4. "A Model for Ferroelectric Phase Shifters," R. Romanofsky and A. Qureshi, IEEE Trans. Magnetics, Vol. 36, No. 5, pp. 3491-3494, September, 2000.

5. "Ka-Band Phased Array Antennas Based on Ba0.60Sr0.40TiO3 Thin-Film Phase Shifters, R. Romanofsky, J. Bernhard, F. VanKeuls, et al., IEEE Trans. Microwave Theory Tech., Vol. 48, No. 12, pp. 2504-2510, December, 2000.

\section{BIOGRAPHY}

Irene Bibyk is the Space Communication and Data Systems Manager at GRC. She has B.S. and M.S. degrees in electrical engineering and applied physics from Case Western Reserve University. Since 1989, she has worked on spaceflight hardware development and operations at NASA, which includes the ST-5 (Space Technology - 5) at Goddard Space Flight Center, Extravehicular Activity at Johnson Space Center, and the Microgravity Experiments at GRC. In these roles, she has primarily developed new technology ground systems for automated operations and experiment modules for Shuttle flights. Ms. Bibyk is also a graduate of MIT's Advanced Study Program through NASA's highly competitive Fellowship Program.

Dr. Robert Romanofsky received the B.S.E.E. from the Pennsylvania State University, the M.S.E.E. from the University of Toledo, and his Doctorate in Electrical Engineering from Cleveland State University (CSU). From 1990-1991, he served as Program Manager for Superconductivity and RF Communications at NASA Headquarters in Washington, D.C., and subsequently served a three month collateral assignment with the White House Office of Science and Technology Policy. He has authored or co-authored over 75 refereed publications in the fields of microwave device technology, high-temperature superconductivity, and microwave applications of thin ferroelectric films. He is a senior member of the IEEE, holds five patents, and is a recipient of NASA's Exceptional Service Medal and the Federal Executive Board "Wings of Excellence" award. $\mathrm{He}$ is currently a member of the Antenna, Microwave and Optical Systems Branch at GRC. Additionally, he is a part-time professor at CSU. 